\title{
XXI.
}

Aus der chirurgischen Abtheilung des Kölner Bürgerhospitals

(Prof. Bardenheuer).

\section{Beitrag zur Casuistik der subphrenischen Abscesse und der Querresection der Niere.}

\author{
Dr. K. Cramer, \\ Assistenzarzt.
}

Von

(Mit einer Abbildung im Text.)

1. Zwei seltenere subphrenische Abscesse.

Subphrenische Abscesse in Anschluss: an eine Milzeiterung sind äusserst selten beobachtet und noch seltener mit Glück operirt worden. Ich lasse deshalb einen derartigen Fall aus der Casuistik Bardenheuer's folgen und knüpfe daran einige Bemerkungen über die Pathologie und Therapie dieser Eiterung.

Herr B. aus K. A namnese: Vor 12 Jahren litt er an starker Stuhlverstopfung, es erfolgte damals 8 Tage lang kein Stuhlgang. In den nächsten Jahren klagte er ab und zu über leichte Schmerzen in der linken Seite und gebrauchte fast fortwährend Abführmittel. 1890 bemerkte der behandelnde Arzt in der Gegend unter dem linken Rippenbogen eine Anschwellung (Koth?).

Am 4. April 1894 erkrankte er mit Schmerzen unterhalb des linken Rippenbogens, Opstipation und hohem Fieber. Der behandelnde Arzt dachte an eine Eiterung in der Nierengegend und an eine Darmentzïndung. Der Patient lag dann 5 Wochen zu Bett. Anfangs Mai im selben Jahre wurde eine Pleuritis diagnosticirt. Husten war nie vorhanden.

Am 15. Juli begannen Schuittelfröste und hohes Fieber, 40-41,8. Er klagte hauptsächlich über Schmerzen in der linken Seite, von der linken Lumbalgegend bis zur Achselhöhle, woselbst eine Dämpfung bis zur Scapula nachzuweisen war. Er bot jetzt das typische Bild des Typhus abdominalis mit dem charakteristischen Fieber. Die Behandlung war eine bei Typhus übliche. Mitte September war der Typhus abgelaufen; der Pat. war fieberfrei und 6 Wochen lang scheinbar gesund. Vom 20. October bis 20. November bestand wieder stets hohes hektisches Fieber bis $39^{0}$, heftige Schmerzen in der linken Seite von der Lumbalgegend ausstrahlend 
bis in die linke Schulter. Eine eitrige Rippenfellentziindung konnte durch den objectiven Lungenbefund ausgeschlossen werden.

Während dieses letzten Termins war stets über der hinteren linken Thoraxhälfte bis zur Scapula eine Dämpfung vorhanden, diese Dämpfung ging allmählich bis 3 Finger breit unter die Seapula zurück.

Status praesens am 20. November 1894. Stark abgemagerter Mann. Ueber der hinteren linken Thoraxhälfte absolute Dämpfung bis 3 Finger breit unterhalb der Scapula. Ueber der Dämpfung kein Athmungsgeräusch. Oberhalb der Dämpfung Vesiculär-Athmen.

Die Milz ist 3-4 Finger breit unterhalb des Rippenbogens zu fühlen. Mit der palpirenden Hand kann man zwischen Milz und Rippenbogen eindringen. Beim sitzenden Patienten geht die eben beschriebene Dämpfung bis zur Wirbelsäule. Legt man ihn auf die linke Seite, so entsteht eine 3 Finger breite Zone, links neben der Wirbelsäule, mit hellem Schall. Die Haut unterhalb des linken Rippenbogens ist in keinerlei Weise verändert, nicht geröthet, nicht infiltrirt. Die Temperatur wechselte in den letzten Wochen, bald mehrere Tage hohes Fieber, 38,5-39, bald einige Tage niedrige Temperaturen. Eine Probepunction zwischen achter und neunter Rippe in der linken Axillarlinie entleert Eiter. Die eingestochene Kanüle geht mit der In- und Exspiration auf und ab.

Diagnose: Abscess, wahrscheinlich unterhalb des Diaphragma oberhalb der Milz.

Operation am 20. November 1894. Einschnitt zwischen 8. und 9. Rippe links in der Axillarlinie, da wo die Probepunction Eiter ergeben hatte. Die $Z$ wischenrippenweichtheile wurden durchtrennt bis auf die Pleura. Man hört deutlich, wie bei der Inspiration Luft durch die Punctionsöffuung aspirirt wird. Ferner kann man durch die gesunde Pleura hindurch die Lungen auf- und absteigen sehen. Die Gesundheit der Pleura ist also nachgewiesen.

Daher sofortige Tamponade dieser Zwischenrippen-Incision und Vernähung der Weichtheile ïber dem Tampon.

Es wird jetzt links der hintere explorative Thürflugelschnitt angelegt. Nach Zurickklappen des Weichtheillapens findet man eine grosse Eiterhöhle unterbalb des Zwerchfells. Die Milz ist ums Doppelte vergrössert, ihre obere äussere Fläche ist mit dem Zwerchfell fest verwachsen. An dieser Stelle der Milz fühlt und sieht man in dem oberen Theil der Milz eine gänseeigrosse, rundliche, prall gespannte fluctuirende Geschwulst, welche auf Einschnitt Eiter entleert. Nach dem Abfluss des Eiters sieht man die Abscesshöhe, sie ist glattrandig, rundlich. Es liegt also nach Anlegen des Thürflügelschnittes zuerst die grosse subphrenische Eiterhöhle vor und dahinter die Milz-Abscesshöhle. Diese beiden AbscessRäume werden mit Jodoformgaze tamponirt. Im Eiter war keine Gasbeimengung; es fanden sich darin bei der mikroskopischen Untersuchung Stäbchen, ähnlich den Typhusbacillen. Gelatine wurde nicht verflüssigt. Auf Kartoffeln wächst die Cultur ähnlich wie Typhusbacillen.

Am 27. November erster Verbandswechsel. Die subphrenische Abscesshöhle hat sich bedeutend verkleinert. Die Milz-Abscesshöhle ist noch deutlich zu sehen. Der Tampon in der Zwischenrippen-Incision wird 
entfernt. Neue Tamponade der grossen Abscesshöhle. Die Wunde granulirte dann langsam aus; der Patient ist $z$. Z. völlig gesund.

In diesem Falle war die differentielle Diagnose nicht ganz leicht und nicht mit Sicherheit zu stellen wegen der eigenthümlichen Anamnese. Schon Anfangs April bestand hohes Fieber und Schmerz in der linken Abdominal-Gegend. Anfangs Mai waren die Symptome einer Pleuritis vorwiegend. Im Juli bot der Kranke das typische Bild eines Typhus abdominalis. Mitte September bis Mitte October war der Kranke scheinbar gesund, um dann im November wieder sehwer zu erkranken unter den Zeichen einer subdiaphragmatischen Eiterung.

Wahrscheinlich war die Erkrankung im April und Mai schon eine Darmentzìndung, vielleicht typhöser Natur, die im Juli recidivirte. Der Typhus läuft $\mathrm{ab}$ und es etablirt sich nach Infarct ein Milzabscess, der während der fieberfreien Zeit im September und October ins linke Subphrenium durchbrach und nun die Zeichen des subdiaphragmatischen Abscesses bot.

Bei der Präcisirung der differentiellen Diagnose, Abscessus Hypophrenicus, kam hauptsächlich in Betracht der 'Tiefstand der Milz, die Dämpfung über den unteren Partieen der linken Thoraxhälfte und dann auch hauptsächlich das mit der In- und Exspiration beobachtete Auf- und Absteigen der eingestossenen Hohlnadel in Verbindung mit dem aspirirten Eiter, oder mit anderen Worten, die Verschieblichkeit des Abscesses und die Beweglichkeit der vergrösserten Milz. Auch wenn die Probepunction keinen Eiter ergeben hätte, musste man eine Supuration unter dem linken Zwerchfell vermuthen. Den Ausgangspunkt des Eiters wagte man bei Aufstellung der differentiellen Diagnose noch nicht zu bestimmen. Er konnte auch von verschiedenen anderen Organen, von Darm, Magen, Nieren, Pankreas ausgehen. Die Lämpfung tiber der hinteren unteren Thoraxhälfte links ist von Lauenstein, Kernig und Parzewsky bei Aufstellung der Diagnose Milzabscess verwendet worden. Speciell Lauenstein fand bei solchen Abscessen Hochstand und Fixation der linken Seite des Zwerchfells.

Zunächst musste dieses Symptom auf eine Eiteransammlung im linken subphrenischen Thoraxraum lindeuten.

Milzabscesse sind im Grossen und Ganzen selten zur Operation gekommen.

Sie entstehen ja bekanntlich nach Rupturen und dann auch besonders im Gefolge von Infections-Krankheiten, so nach Typhus abdominalis, Recurrens, Pyämie, Gelenkrheumatismus. Kernig fand bei 400 Recurrensfällen fünfmal, Petrowsky bei 357 Fällen derselben Infectionskrankheit, dreimal Milzabscesse. Weiter können Eiterungen in der Milz auftreten, nach Verletzungen und durch Uebergreifen von Eiterungen in der Naihe liegender Organe. Der Vereiterung geht meist eine nekrotisirende Bacterienansiedlung oder Circulationsstörung voraus. Die Abscesse sind von Nuss- bis Hühnereigrösse mit glatten Wandungen und finden sich häufiger im oberen Theil der Milz. 
Diffuse Vereiterung mit multiplen Abscessen scheint seltener zu sein.

Einen derartigen Fall erwähnt Herrlich. Nach Rheumatismus waren mehrfache Abscesse in der Milz entstanden und hatten einen subdiaphragmatischen Abscess erzeugt.

Ich komme auf diesen Fall noch zurück. Der oder die Abscesse können Monate und Jahre lang, ja sogar manchmal für immer symptomlos bleiben und schliesslich verkalken. Sie können aber auch sehr rasch zum Tode führen.

Wobl am häufigsten kommt es zu Perforationen in die Nachbarschaft, der meist eine Perisplenitis voraufgeht.

Es sind Durchbrüche beschrieben worden in den Magen (Coz è und Fahner) Dickdarm (Herrmann, Brown und andere) durch das Zwerchfell (Vid a l, K er nig, Ma n tell). Ferner sind Fälle bekannt, wo die Milz mit den Bauchdecken verwachsen war (Heide, Fahner, Barbieri). Die Operation ist bei diesen letzteren Fällen nicht schwer, die Bauchwand ist dann infiltrirt, geröthet und es wird nicht allzuviele Schwierigkeiten machen, den Abscess extraperitoneal nach Durehschneidung der Bauchdecken, zu finden. In unserem Falle waren keine Merkmale an den Bauchdecken zu finden, die auf einen naheliegenden Abscess hätten hindeuten können. Keine Röthung, keine Schwellung, keine Infiltration. Den schon erwähnten He r rli ch'schen und den $\mathrm{Now}$ ak'schen Fall finde ich als die einzigen, wo Milzabscesse einen subphrenischen Abscess hervorbrachten. Es wurden ab und zu perforirte Milzabscesse mit eitriger Perisplenitis operirt, eine typische subphrenische Eiterung im Anschluss an einen Milzabscess und gliucklicher operativer Therapie, scheint man ganz extrem selten beschrieben zu haben.

Die einschlägigen Fälle von $\mathrm{H}$ er r lich und $\mathrm{N}$ ow ak sind Leichenbefunde.

Häufiger sind metastatische Abscesse mit Bildung eines hypophrenischen Abscesses in der Leber beobachtet worden, besonders nach tropischen Lebererkrankungen. Interessant ist in unserem Falle noch das Verhältniss der Lunge zum Pleurasack. Es wirde nach der Punction beim Einschnitt zwischen achter und neunter Rippe anf den Pleurasack Luft aspirirt, ein Beweis dafür, dass der Pleuraraum selbst keine Flitssigkeit enthielt.

Bekanntlich kommt es ja häufig bei derartig länger bestehenden subphrenischen Eiterungen zu einem Exsudat in dem betreffenden Pleuraraum auch ohne Perforation des Zwerchfelles. Das Exsudat kann serös, serös eitriger, oder jauchiger Natur sein, ja es wurden 
sogar Fälle beobachtet, wo noch nachträglich nach Eröffnung des lienalen und perisplenitischen Abscesses eine eitrige Pleuritis eintrat.

Die Therapie des Milzabscesses und der davon ausgehenden Eiteransammlung im Subphrenium wird eine verschiedene sein können. Sie soll dem Eiter-Abfluss nach aussen verschaffen, die Abscesshöhlen zur endgültigen Ausheilung bringen und eine allgemeine Peritonitis vermeiden. Eine Punction in Verbindung mit Aspiration wird nur in sehr seltnen Fällen zum Ziele führen. Häufig wird man mit einer grossen Incision auskommen.

Den von Lauenstein angegebenen Weg, die Probepunctionskanüle stecken zu lassen und an ihr entlang auf den Abscess vorzudringen, kann man nicht immer einschlagen. Die Kanuile kann, sowohl bei lienalem, als auch subphrenischem Abscess, zunächst die gesunde Pleura durchdringen, wie in unserem Falle, und dann erst den Eiter treffen. Man würde so unnöthigerweise den Thorax eröffnen und der Entwickelung eines Pyopneumothorax Vorschub leisten. Ein Explorativschnitt wird hier mehr am Platze sein. Der hintere Thürflügelschnitt hat den Vortheil, dass man von ihm aus extraperitoneal die gewünschte Gegend genauer abtasten, sich iiber die Lage des Abscesses besser orientiren und alle nöthigen Eingriffe an der Milz ausführen kann. Bardenheuer hatte auch anfänglich die Absicht, von einem hinteren Thürflügelschnitte aus vorzugehen.

Da indess die Diagnose keine ganz sichere war, so entschloss er sich, zuerst die äussere Fläche der Pleura costalis frei zu legen, um sich zu überzeugen, ob dieselbe verdickt war und ob nicht ein abgesacktes pleuritisches Exsudat dort vorlag. Dieser Eingriff war ein relativ geringer. Man konnte alsdann noch immer den anderen Weg zur Bloslegung des Heerdes einschlagen und den Thürfligelschnitt anlegen. Hätte man die beiden Pleurablätter verwachsen oder ein pleuritisches Exsudat gefunden, so hätte man auch durch Pleura und $Z w e r c h f e l l$ hindurch in den subphrenischen Abscess gelangen können.

Die Milz muss man je nach dem Sitz und der Zahl ihrer Abscesse und der Form und Art der Perisplenitis verschieden behandeln. Einen solitären Milzabscess wird man incidiren und tamponiren. Weiter kann man Stücke aus der Milz ausschneiden oder quer reseciren, wenn einzelne benachbarte Theile des Organs von mehreren Abscessen durehsetzt sind. So vollendete Bard e $n$ h e u er von diesem Schnitte aus eine quere Milzresection wegen einer Cyste und die Totalexstirpation der vergrösserten Milz. Vielleicht wächst ja die Milz auch, wenn nur ein ganz kleines Stück noch übrig geblieben ist, wieder zur normalen 
Grösse an. Endlich kann man die Milz ganz entfernen bei ausgedehnter suppurativer Perisplenitis. Es haben dieses wegen Abscessen Ferrerius und Myers gethan.

Frau Rosa Koch, 36 Jahre alt.

A namnese: Die Patientin bat einmal geboren und einmal abortirt. Vor einem Jahr starker Scheidenausfluss mit Urinbeschwerden.

Eine Untersuchung in Narkose ergab beiderseits neben dem Uterus, diesen umschliessend und mit der Beckenwand leicht verwachsen, entziindliche, mannsfaustgrosse, harte Adnextumoren. Nach der Untersuchung traten Collapserscheinungen auf mit Cyanose und peritonitischen Reizerscheinungen.

Die Temperatur war 40,2, der Puls 156.

In den nächsten Tagen post explorationem ist eine leichte Dämpfung fast über dem ganzen Abdomen nachzuweisen. Nach weiteren 14 Tagen ist der Percussionsschall handbreit oberhalb der Symphyse and den Poupart'schen Bändern gedämpft. Es bestand Brechneigung und Temperatursteigerung bis 39,5. Die Patientin klagte iber Schmerzen in der Lebergegend. In der vergrösserten Leber Dämpfung, Druckschmerz, besonders in den unteren Zwischenrippenräumen. Hinten rechts unten eine handbreite Dämpfung über der Lungengegend, schräg nach der Seite und vorne sinkend, bis sie in der Mammillarlinie die Leberdämpfung erreicht. Ueber dieser Dämpfung war Anfangs Reiben zu hören, später gar kein Athmungsgeräusch mehr. Im Urin war kein Eiweiss. Am 28. December intercurrente Carbolintoxation infolge von Guajacolglycerin-Einreibungen.

Die Temperatur blieb dann unter 38,0. Die subjectiven Beschwerden besserten sich, bis am 11. Januar 1895 eine neue Attacke eintrat mit Athembeschwerden, Schmerzen in der Lebergegend und erhöhten Temperaturen. Eine Punction im 10. Intercostalraum in der hinteren Axillarlinie rechts ergiebt schleimige, eitrige Flissigkeit.

Status am 21. Januar 1895: Temperatur 37,8, Puls 108. Der Orin ist ohne Eiweiss und Zucker; liber der Symphyse und den Poupart'schen Bändern eine handbreite Dämpfung, eine gleiche iber der rechten Lunge hinten unten. Ueber dieser letzteren Dämpfung ist kein Athmungsgeräusch zu hören. Leberdämpfung innerhalb der normalen Grenzen.

Diagnose: Abscessus subphrenicus dexter.

22. Januar Operation. Längsschnitt auf der elften Rippe in der rechten Axillarlinie, Resection eines $8 \mathrm{Cm}$. langen Stïckes dieser Rippe. Beim präparirenden Vorgehen in der Tiefe kommt man durch $1 \mathrm{Cm}$. dickes, hartes, schwieliges Gewebe, nach dessen Durchschneidung im vorderen unteren Wundwinkel Eiter hervorquillt. Es wird nun der Anfangsschnitt nach vorne verläugert und auf ihm der hintere Bardenheuer'sche Thurfllgelschnitt complettiert. Nach Zuriticklappen des Thüflugellappens kann man mit der Hand in eine Abscesshöhle eindringen, die sich zwisehen Zwerchfell und Leber befindet. Das Zwerchfell war nacl oben, die Leber nach vorne gedrängt. Die Niere und das pararenale Gewebe waren intact. Vollständiger Abschluss gegen die tibrige Peritonealhöble. Die untere Hälfte des Thürflugelschnittes wurde genäht, die Abscesshöhle mit Jodoformgaze tamponirt. Aus der weiteren Krankengeschichte ist noch zu 
erwähnen, dass die Wunde sich rasch reinigte und nach 3 Wochen mit Granulationen ausgefillt war.

Der subphrenische Abscess war somit geheilt, aber das ursächliche Moment, die entzündlichen Adnextumoren, blieben. Sie wurden am 5. April 1895 mittelst suprasymphysärem Querschnitt nebst dem Uterus exstirpirt. Im Becken fanden sich ausgedehnte Verwachsungen, durchsetzt von Abscessen. Die Tuben waren ebenfalls hochgradig verändert und vergrössert, ebenso die Ovarien.

Zur Zeit, im September 1895, geht es der Patientin gut, sie hat an Körpergewicht zugenommen, sieht gut aus und hat keinerlei Beschwerden mehr.

Bei dieser Patientin machte die Aufstellung der Diagnose weniger Schwierigkeiten, da der Fall unter unseren Augen im Krankenhause verlief. Im Anschluss an eine Exploration per vaginam entsteht bei einem entzilndlichen Adnextumor durch Austritt von Eiter diffuse Peritonitis. Die Bauchfellentzindung heilt ab. Es bleibt eine Beckenperitonitis zurilck, die aufwärts ihren Weg findet und einen subdiaphragmatischen Abscess erzeugt.

Dafür dass nur eine Beckenperitonitis bestanden hat, sprechen die Verhältnisse des Peritoneums und seiner Organe, die ja bei der zweiten Operation bei der Ausräumung des kleinen Beckens zur Atopsie in vivo kam. Anlässlich dieser zweiten Operation möchte ich bemerken, dass B a r de n b u er den suprasymphysären Querschnitt nur noch höchst selten bei ganz schwierigen Operationen im kleinen Becken anwendet. Durch Trendelenburg'sche Hochlagerung hat man bei den meisten einschlägigen Erkrankungen jetzt nach einem Längsschnitt Platz und Uebersicht genug.

Maydl hat in seinem bekannten Sammelwerk über subphrenische Abscesse nur 4 Fälle derartiger Abscesse erwähnt, die nach Genitaleiterungen und zwar nur bei Frauen entstehen können. Alle 4 Fälle kamen unoperirt zur Section. In dreien dieser Fälle war der Abscess wie der unsrige rechts gelegen. Bei allen vieren, wieder analog dem unsrigen, waren Pleura und Lunge mitbetheiligt. Bei Einzelnen fand man auch sonst noch Abscesse an anderen Stellen des Peritoneums, so besonders in der Milzgegend. Es dürfte also unser Abscess ein besonders typisches Beispiel sein für diejenigen Gruppen subphrenischer Abscesse, die im Anschluss an eine Genitalerkrankung des Weibes entstehen. Der Fall wird noch interessanter durch die nachfolgende Herausnahme des Uterus und der Ausräumung des kleinen Beckens, wobei man das Verhalten der iibrigen Bauchorgane controlliren und soweit dies möglich, normal finden konnte.

\section{Querresection der Niere wegen Tuberculose.}

I. Herr L. aus K., 32 Jahre alt.

A n amnese: In seiner Familie ist keine tuberculöse erbliche Belastung nachzuweisen. Er selbst will immer gesund gewesen sein. An- 
fangs November 1894 erhielt er beim Fahren eines Wagens einen Stoss in die Abdominalgegend, worauf er sein jetziges Leiden zurlickfuhrt. 14 Tage nach diesem Trauma erkrankte er mit hohem Fieber und Schmerzen in der rechten Renalgegend. Der behandelnde Arzt dachte an Senkungsabscess und an Typhlitis.

Eine differentielle Diagnose konnte damals nicht gestellt werden. Am 2. Januar 1895 erfolgte seine Aufnahme ins Krankenhaus. Er hatte damals leichtes Fieber. Meistens war letzteres bektisch, mit abendlichen Steigerungen bis 38,7 . Im Urin war nie Eiweiss. Die Wirbelsäule zeigte nichts Abnormes.

Eine Untersuchung in Narkose kurz nach der Aufnahme ergiebt, dass die Fossa iliaca gefüllt und die Lumbalgegend infiltrirt ist. Die Diagnose schwankt zwischen Paratyphlitis, eiteriger Psoitis und Senkungsabscess, von der Wirbelsäule, vielleicht von einem Querfortsatze der Lendenwirbelsäule ausgehend. Es wird deshalb zwischen letzter Rippe und Darmbeinkamm hinten neben der Wirbelsäule eingeschnitten, ein retroperitonealer Abscess blossgelegt und incidirt. Ein Eitergang führt bis nach der Wirbelsänle, ohne dass an dieser selbst etwas Abnormes hätte gefunden werden können. Eine manuelle Untersuchung von der Incisionswunde aus ergab im Bereiche des Coecums und der Niere ebenfalls keine Abnormitäten. Der Psoas war durch die Jauche vollständig zerfallen. Tamponade der Wundhöhle.

Die Wunde verkleinerte sich im Laufe der nächsten Wochen ziemlich rasch. Die Eiterung bestand aber profus fort. Das Fieber war wechselnd. Bald war er einige Tage fieberfrei, bald traten leichte abendliche Temperatursteigerungen ein.

Im Urin kein Eiweiss und nichts Abnormes. Häufig wurde der Pat. untersucht und die Wunde sondirt. Das ursächliche Moment der Eiterung war nicht $z \mathfrak{u}$ finden.

Ueber den Lungen konnten auch keinerlei Abnormitäten nachgewiesen werden.

Am 12. Mai 1895 wird in der rechten Nierengegend ein Tumor nachgewiesen. Man fühlte in der Nierengegend eine gut faustgrosse, ziemlich harte Resistenz mit stumpfen Rändern. Somit war ein Anhaltspunkt für die Eiterung gegeben.

Operation 16. Mai 1895. Hinterer Bardenheuer'scher Thürflïgelschnitt. Nach Zurückklappen des Weichtheillappens zeigt sich die Niere fast ums Doppelte vergrössert und zwar durch Volumenzunahme des unteren Poles des Organs. Das Peritoneum wird an der vorderen Seite der Niere absichtlich etwas eröffnet, zur Ermöglichung der directen Palpation der Niere. Der Operateur (Bardenhe u er) kann auf diese Weise feststellen, dass die Consistenz der anderen Niere die gewöhnliche ist und speciell, dass sich keine Buckel an ihr befinden und sich keine Fluctuation oder Vergrösserung zeigt. Die Operation wird hierdurch nicht lange aufgehalten.

Sofort wird die Incisionsöffnung im Peritoneum wieder genälit, die Niere aus ilirer Capsula adiposa ansgehuilst und diese Kapsel zur Sicherung der Peritonealnaht über diese an die benachbarten Weichtheile angenäht. Es besteht keine Para- oder Perinephritis im eigentlichen Sinne 
des Wortes. Man kann jetzt an der luxirten Niere deutlich sehen, dass der untere Pol der Niere stark vergrössert ist. Dieser Theil der Niere sieht hellgelblich aus und ist mit noch helleren, kleinen Knöteben besetzt, von Hirse- bis Erbsengrösse. Die obere Hälfte der Niere zeigt keine Veränderung. Der erkrankte untere Pol grenzt sich ziemlich scharf durch seine Farbe und seine kugelige Gestalt, von der oberen gesunden Partie ab. Die nntere Hälfte der Niere wird durch einen nach dem Nierenbecken zugehenden, senkrecht zur Längsaxe des Organs gesehenen grossen Schnitt abgeschnitten. Die Blutnng ist gering und kann leicht durch temporäre Tamponade

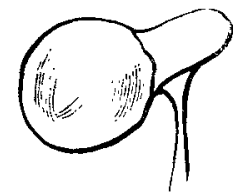
und einige Umstechungen beherrscht werden. Im eröfneten Nierenbecken sieht man gesunde Schleimhaut. Der Puls war am Schluss der Operation, die 25 Minuten danerte, selır klein geworden. Tamponade der Wundhöhle mit Jodoformgaze. Verband. Starker Collaps. Der Patient starb 5 Stunden post operationem, ohne sich von dem Eingriff erholt zu haben. Es war das letzte Mittel, das versucht werden musste, den dureh die lange andauernde Eiterung sehr geschwächten Patienten zu retten.

Vielleicht hat sich nach dem Trauma, dem Stoss in die Nierengegend, ein schon bestehendes kleines tuberculöses Abscesschen durch die Nierenkapsel ins retroperitoneale Gewebe ergossen und zur Entwicklung der Psoitis geführt. Bei der ersten Operation, der Eröffnung des psoitischen Abscesses, war die Niere nicht vergrössert.

Während der Behandlung dieses letzteren Abscesses, machte dann die Tuberculose der Niere Fortschritte, verbreitete sich. Es ist auch möglich, und dieses scheint mir als das Wahrscheinlichere, dass wir es mit zwei verschiedenen Processen zu thun hatten; mit einer eiterigen Psoitis neben einer Nierentuberculose. Diese tuberculösen Processe in der Niere befanden sich zur Zeit des Traumas im Anfangsstadium und gelangten daun vielleicht durch den Stoss und die damit verbundene Quetschung der Niere zur Propagation. Eine Section wurde nicht gestattet, die mikroskopische Untersuchung des Präparates bestätigte die makroskopische Diagnose: Tuberculose.

11. Arenz aus K., 18 Jalire alt, Modelleur.

Anamnese: Er war bis zum 10. Jahr immer kränklich. Dreimal war Lungenspitzenkatarrh nachzuweisen. Im Winter 1894-95 klagte er mehrfach über Schmerzen in der linken Seite und legte sich auch dieserhalb häufig zu Bett. Im April 1895 erkrankte er mit Leibschmerzen und Aufstossen. Bald darauf traten Schmerzen in der linken Nierengegend aư', starke Diarrhöen und Rückenschmerz. Es bestand Fieber bis 39,5. Ab und zu klagte er auch uber Schmerzen in der rechten Seite. Obstipation wechselte mit Durchfall ab. Der Urin war eiweissfrei, die Urinmenge reichlich.

Status am 12. Mai 1895: Magerer Patient. Die Haut uber der linken Lumbalgegend ist geröthet. Diese Weichtheile der Nierengegend 
fühlen sich teigig an; darunter deutlich Fluctuation, die vom Rippenbogen bis zum Darmbeinkamm reicht. Ueber den Lungen nichts Abnormes. Urin frei von Eiweiss und Zucker.

Diagnose: Abscessus perinephriticus.

Operation: 13. Mai 1895. Hinterer Bardenheuer'scher Thürflügelschnitt. Es präsentirt sich ein grosser Abscess, der von der 12. Rippe bis zur Fossa iliaca reicht. Der Eiter ist gelblich, dickftissig. Die Capsula adiposa ist zum Theil mit der Capsula fibrosa verwachsen. Der Abscess ist mehr para- als perinephritisch gelegen.

Nach dem Loslösen der fibrösen Kapsel kann man deutlich sehen, dass der obere Theil der Drüse von normaler Gestalt und Farbe ist. Der untere Theil ist leicht gelblich verfärbt und etwas verdickt. Im unteren Pol einige disseminirte, kleine, gelbliche Stellen, die beim Einschneiden Eiter entleerten.

Es wird nun die unterste Kuppe der Niere in $1 / 2 \mathrm{Cm}$. Dicke quer abgeschnitten. Dann wird diesem Schnitte parallel ein zweiter geführt, ebenfalls von der Dicke eines halben Centimeter. Diese abgeschnittenen Scheiben sind durchsetzt von kleineren und grösseren Herden, deren maximale Grösse die einer Erbse ist. Sie enthalten dicken, weisslichgelben Eiter. Weiter wird nun fast die ganze untere Hälfte der Niere, ähnlich wie man eine Ananas in Scheiben zerlegt, durch Querschnitte langsam entfernt. Bardenheuer hört mit diesen quer resecirenden Schnitten auf, sowie sich keine Herde, sondern nur noch gesundes Nierengewebe auf der Schnittfäche zeigt. Das Nierenbecken wird angeschnitten. Man kann seine Innenfläche als gesund deutlich taberblicken. Die Blutung ist nicht nennenswerth. Die Nierenwundfläche ebenso wie die grosse Abscesshöhle wird tamponirt.

Aus dem Heilungs- und Wundverlauf ist hervorzuheben, dass der Verband in den ersten Wochen täglich gewechselt wurde und sehr rasch mit Urin durchtränkt war. Anfangs Juni wird die urinöse Durehtränkung des Verbandes immer geringer. Vom 10. Juni ab ist kein Urin im Verband mehr zu bemerken. Es bestand kein Fieber post operationem.

Die gut granulirende Wunde wurde nach Aufhören des Urinausflusses mit Heftpflaster zusammengezogen und heilte rasch ohne $Z$ wischenfall zu. Anfangs September 1895 sieht der Operirte gesund aus, hat an Körpergewicht zugenommen und ist wieder vollkommen arbeitsfähig. Der Urin ist frei von Eiweiss, die Wunde ist völlig geschlossen geblieben. Auch hier ergab die mikroskopische Untersuchung des Präparates Tuberculose.

Bardenheuer (Köln) liess im Jahre 1887 durch Franz Bardenheuer an Hunden experimentelle Untersuchungen vornehmen tiber die Zulässigkeit und Möglichkeit von partieller Resection und die Regenerationsfähigkeit des Nierengewebes. Franz Bardenheuer wies damals nach, dass man bei Hunden sehr wohl Stticke einer Niere ohne besondere Störung des Allgemeinbefindens und ohne directe Operationsgefahr entfernen kann, und dass die so behandelte Drise das verlorene Gewebe durch Hyperplasie zu ersetzen sucht 
und wirklich ersetzt. Die Uebertragung dieser Versuche auf den Menschen legte die Kenntniss von der Nierenruptur nahe.

Dass damals, vor jetzt 8 Jahren, noch keine Resection der Niere am Menschen ausgeführt wurde, lag wobl hauptsächlich an dem in jeder Zeit bestehenden Enthusiasmus über das Gelingen der totalen Nephrectomie und an dem Mangel an Erfabrungen in der Nierenchirurgie als solcher. Czerny liess bald darauf einen Fall von Herczel beschreiben, bei welehem eine mit bröckligem Brei angefulltte Höhle des Nierengewebes eingeschnitten, ausgelöffelt, ausgeräumt und die Abscesshöhle eliptisch umschnitten wurde.

1889 erschien die experimentelle Arbeit $T$ u f fi er's, welcher ebenfalls das Gelingen partieller Nierenresectionen und die Regenerationsfähigkeit des Nierengewebes an Thieren nachwies. Er fand nach bestimmter Zeit die partiell entfernte Nierensubstanz, durch Hyperplasie und Neubildung von Glomerulis ersetzt. Er rieth auch das Nierenbecken nicht anzuschneiden zur Vermeidung der gefürchteten Nieren-Bauchdecken-Urinfistel.

1890 referirte $\mathrm{K} \ddot{u} \mathrm{~m} \mathrm{~m}$ el über 2 Fälle von partieller Nierenresection.

In dem einen Falle entfernte er ein Drittel der Niere, in dem anderen die obere Kuppe. 1891 publicirte Bardenheuer 2 Fälle von querer Nierenresection. Weiter ist mir noch ein Fall bekannt von D'Antona, der wegen einer Cyste den oberen Pol der Niere quer abschnitt. Alle diese Fälle beweisen, dass man Stücke der Niere entfernen kann, dass sich keine dauernde Nierenbauchdeckenfistel einzustellen braucht, und dass man die dabei auftretende gefurchtete Blutung in der Gewalt bat. Ueber derartige Operationen wegen Tuberculose der Niere habe ich in der Literatur nichts gefunden. Vielleicht handelt es sich in dem einen oder anderen Fall um Tuberculose, allein betont finde ich diese Indication zur partiellen Nierenresection nicht.

Soll man bei Tuberculose der Niere die Querresection machen, ist diese Operation überhaupt als Erfolg versprechend zulässig, oder soll man nephrectomiren?

Diese Fragen sind nicht leicht und nicht ohne Weiteres zu beantworten.

Es kann sich bei partiellen Resectionen tuberculöser Nieren wohl nur um die hämatogene, die häufigere Form der Tuberculose handeln. Auf die Frage nach dem Vorbandensein der anderen Niere, möchte ich hier nicht eingehen und von der anderen Form der Nierentuberenlose der vom Nebenhoden, Samenleiter, Prostata, Samenbläschen und Harnleiter aufsteigenden, jetzt absehen. 
Die hämatogene Localtuberculose der Niere beginnt ja bekanntlich da, wo der Blutstrom Tuberkelbacillen zur Ansiedlung bringt. Sie ist häufig auf das Gebiet eines Astes der Nierenarterien beschränkt, miliar (Zi e gle r).

Kommt der Infectionsherd nicht zur Ausheilung, so entsteht ein Erweichungsherd, der ja schliesslich die ganze Niere in Mitleidenschaft ziehen und in einen Brei verwandeln kann. Zwischen dieser letzteren grössten Zerstörung der Phthysis renum und der Anschwemmung der Tuberkelbacillen, liegen natürlich alle möglichen Zwischenund Uebergangsformen. Ich möchte sie hier nur erwähnt haben. Es kann ja auch die ganze Niere, nicht nur ein Theil von Pilzen tiberschwemmt werden; es können sich Steinbildungen anschliessen; die Niere kann durchsetzt sein von grösseren und kleineren Herden u. s. w.

Die doppelseitige Erkrankung beider Nieren an hämatogener Tuberculose dïrfte nicht so häufig sein, wie man noch vor $7-10$ Jahren annahm. Fand doch Palet bei 100 Nephrectomien Nierentuberculöser nur 16 mal eine beidseitige Erkrankung. Auch Bar denh e u er betont schon 1887, dass die häufigere Form der Nierentuberculose, die hämatogene, meist einseitig sei. Seine damalige Behauptung hat er sich in den letzten 8 Jahren bestätigen sehen, theils durch das Gesundbleiben Nephrectomirter, theils durch Sectionsbefunde. Es wäre demnach die Herausnahme der ganzen Niere bei hämatogener Tuberculose eine berechtigte Operation. Um so mebr, da man annehmen darf, dass in vielen Fällen von doppelseitiger Erkrankung an hämatogener Nierentuberculose, die im Körper belassene ebenfalls erkrankte Niere, nach Entfernung der einen, leichter zur Abheilung kommen wird; ähnlich wie bei gleichzeitiger tuberculöser Lungenspitzenaffection und ausgedehnter Gelenktuberculose eines Gliedes, nach Amputation des letzteren in vielen Fällen die progressive Erkrankung der Lunge regressiv wird und vielleicht abheilt.

Die Nephrectomie wird also am Platze sein, wenn die bämatogene Tuberculose den grössten Theil der Drüse diffus zerstört hat, wenn das Nierenbecken schon mit afficirt ist und man nicht weiss, wie weit auf dem Ureter der Process sich schon erstreckt.

Ist der Ureter miterkrankt, so muss man ja gleichzeitig diesem nachgehen und ihn, soweit die Infection reicht, mit entfernen.

Thut man dieses nicht, so setzt man sich der Gefahr aus, dass sich eine Ureter-Bauchdecken-Fistel etablirt, welche nie zuheilt.

Allein wie unsere beiden Fälle zeigen, giebt es auch Formen von Nieren-Tuberculose, wo man sehr gut einen grösseren oder kleineren 
Theil dem Organismus erhalten kann in der Hoffnung, dass sich von dem zurückgelassenen Theil aus das Organ regenerirt. Vielleicht ist diese Nierentuberculose nicht allu selten, kommt aber nicht immer zur Diagnose.

Man darf wohl annehmen, dass die Tuberkelerreger hier durch eine dem unteren Pol der Niere angehörige Arterie angeschwemmt wurden und deshalb nur in dieser Gegend der Drüse ihre zerstörende Wirkung entfalten konnten.

In dem einen Falle (A r e nz) war ja auch ein Lungenspitzenkatarrh nachgewiesen worden.

Bei beiden Patienten bestätigte die mikroskopische Untersuchung die bei Freilegung der Niere gestellte Diagnose: Tuberculosis renum partialis.

Bei dem gestorbenen Kranken wurde die andere Niere durch directe Palpation mit der das Peritoneum traversirenden Hand als gesund nachgewiesen. Es ist dieses natürlich mit annäherender Sicherheit nur einem geübten Nierenoperateur möglich. In dem Falle Arenz kam diese Untersuchungsmethode nicht zur Anwendung. Sie musste unterbleiben, weil ja sonst die Peritonealhöhle mit dem Eiter des paranephritischen Abscesses inficirt worden wäre. Indess scheint mir auch hier die Gesundheit der anderen Niere durch das jetzige blühende Aussehen, die Zunahme des Körpergewichtes dem eiweissfreien Urin und die völlige Arbeitsfähigkeit nachgewiesen zu sein. Das Nierenbecken konnte in beiden Fällen durch Ocularinspection als gesund erkannt werden.

Ich glaube demnach annehmen zu durfen, dass es sich in beiden Fällen um isolirte, bämatogene Tubereulose in der unteren Hälfte nur einer Niere handelte und dass man zweckmässig bei dieser Form der Tuberculose die Querresection macht und nicht das ganze Organ nephrectomirt. Selbstverständlich wird man auch zuweilen, bei sehr begrenzter Tuberculose in die Lage kommen können, nur die kleinen Herde auszuschneiden.

Jedenfalls dürfte es vorläufig bei ausgedehnter Querresection noch immer indicirt sein, das Nierenbecken za eröffnen, und sich von der Beschaffenheit seiner Schleimbaut zu tiberzeugen, selbst wenn man weiss, dass das Ausfliessen von Urin beim uneröffneten Nierenbecken fast gar nicht stattfindet. 